\title{
A Novel Simulator Of Radar Clutter Environment
}

\author{
Zhiyong LIU ${ }^{*}$, Yuying LIANG ${ }^{\dagger}$ \\ Department of Electronics and Optics Engineering, Machanical Engineering College, Shijiazhuang \\ 050003, China
}

Keywords: Radar Clutter, Reverberation Chamber, Spectrum Model, Doppler Spread

\begin{abstract}
In this paper, the characteristic of radar clutter spectrum is studied in reverberation chamber (RC). The similarity between reverberation chamber field environment and radar clutter environment and the feasibility of simulating radar clutter environment in reverberation chamber have been analysed. A novel spectrum model is proposed, which establishes a bridge between reverberation chamber and radar clutter. It is validated that the model is well fitted with the measurement data in RC. This paper tests and verifies the possibility of simulating radar clutter environment in RC and can be taken as the reference for radar clutter simulation in $\mathrm{RC}$ in the future.
\end{abstract}

\section{Introduction}

Radar clutter is an important part of radar battlefield electromagnetic environment, the existence of radar clutter will seriously affect the radar detection performance [2], simulating radar clutter accurately has important significance to the design of radar system and detection performance of radar. Clutter simulation method is mainly divided into two aspects: clutter signal simulation and clutter environment simulation. Clutter signal is simulated mainly by computer simulation software and the physical or semi physical clutter simulator, which is applied by injecting way; radar clutter environment is simulated mainly in the open field, anechoic chamber and reverberation chamber; the former can simulate complex time domain and frequency characteristics of the clutter signal accurately, but lack the authenticity of the scene. The open field simulation experiment is always affected by too many factors such as weather, temperature, humidity and so on. Meanwhile, it will cost much time and cost; the manufacturing process of anechoic chamber is complex and the cost is too high; the reverberation chamber has the possibility of simulating clutter in multi dimension. Good shielding performance, simple manufacturing process, and low cost because no microwave absorbing materials is used [3]. All above makes it an advantage to choose the RC as the simulator of radar clutter.

\section{Reverberation chamber}

A Reverberation Chamber (RC) is a high-quality factor of the metal shield wall, mainly consist of the stirrers, antenna and the AUT. In the reverberation chamber, the boundary condition of the reverberation chamber is changed continuously to build a statistically uniform field environment by the continuous or step stirring of the stirrer. [4].

The electric field $\vec{E}$ at location $\vec{r}$ in a source-free, finite volume can be represented as an integral of plane waves over all real angles [5]:

$$
\vec{E}(\vec{r})=\iint_{4 \pi} F(\vec{\Omega}) \exp (\overrightarrow{i k} \bullet \vec{r}) \mathrm{d} \Omega
$$

The mean value of the electric field $\langle\vec{E}(\vec{r})\rangle$, which can be derived as (2):

$$
\begin{aligned}
& \langle\vec{E}(\vec{r})\rangle=\left\langle\iint_{4 \pi} F(\vec{\Omega}) \exp (i \vec{k} \bullet \vec{r}) \mathrm{d} \Omega\right\rangle \\
& =0 \\
& \left\langle|\vec{E}(\vec{r})|^{2}\right\rangle=4 C \iint_{4 \pi} d \Omega=16 \pi C \equiv E_{0}^{2}
\end{aligned}
$$

The mean-square value of the electric field is $E_{0}^{2}$, which is depend on position; 


$$
\left\langle\left|E_{x}\right|^{2}\right\rangle=\left\langle\left|E_{y}\right|^{2}\right\rangle=\left\langle\left|E_{z}\right|^{2}\right\rangle=\frac{16}{3} \pi C \equiv \frac{E_{0}^{2}}{3}
$$

Formula (2) and (3) reflect the uniformity property of the electric field inside the RC and formula (4) shows the isotropy property of an idealized reverberation chamber. Resemble properties can be obtain for the magnetic field and its rectangular components [6].

\section{Feasibility analysis}

The reverberation chamber is an electrically large cavity, the boundary conditions of the reverberation chamber will change with continuous rotation of the stirrers, there is a statistically homogeneous, isotropic, random polarization field environment for a well stirred reverberation chamber. From the angle of signal transmission, mechanism of production of field environment in RC and radar clutter is resemble. The existence of the stirrers increases the complexity of reverberation chamber structure and it will generate a Random time-varying field environment [7]. When signals are sent into RC, a multipath environment is eventually formed in the reverberation chamber due to the reflect of wall and the stirrer, the received response of reverberation chamber is actually the sum of different amplitude, different phase, different polarization signal superposition. For radar clutter, antenna transmit electromagnetic signal to the environment, because of the complexity of the environment. There are a variety of moving and stationary scatters with different shapes, transmission environment of radar signal is a multipath environment, the received signal is the superposition of a variety of vector signal. Thus, it is possible to simulate radar clutter in RC.

\section{Spectrum model}

The radar clutter power spectrum model can be considered as the radar Gauss spectra in early. However, With the improvement of radar precision, gauss spectra have not been good fit to the clutter power spectrum. Then the power-law spectrum was supposed to describe clutter power spectrum, but it is a serious issue that decay rate is different at high power levels and low power levels for power-law spectrum. The exponential spectrum can be used to describe the clutter power spectrum model in a very high dynamic range, a new exponential spectrum which has been used to describe the clutter of windblown vegetation was proposed in [1]:

$$
P_{t o l}(f)=\frac{r}{1+r} \delta(f)+\frac{1}{1+r} P_{a c}(f),-\infty<f<\infty
$$

The right of formula can be divided into two parts, the first part is the DC part of the clutter spectrum of return signal where the radar pulse is backscattered by the static scatters. The second part is the AC part, where the radar pulse is backscattered by the moving scatters. The two can be expressed with the Dirac delta function and exponential function respectively.

$$
P_{a c}(f)=\frac{\lambda \beta}{4} \exp \left(-\frac{\lambda \beta}{2}|f|\right),-\infty<f<\infty
$$

Where $\lambda$ is the radar signal wavelength and $\beta$ is the shape parameter which is a function of the wind conditions, and the spectrum width is proportional to wind speed.

Previous research on field environment of reverberation chamber show that the spectral characteristics of the environment mainly has relationship with the stirring velocity and signal frequency, but all studies are still remain at qualitative level. Then, it is found that the spectral model of reverberation chamber is exponential with the deep research of reverberation chamber.

For the continuous stirred reverberation chamber, the continuous stirring of the stirrers can be regarded as the motion of the scatters of the radar and the wall of RC can be regarded as stationary scatters in the face of the radar signal [9]. Therefore, the above model can be used to describe the spectral characteristics of reverberation chamber, in other words, the reverberation chamber can be a simulator of radar clutter spectra because the above model has been verified that it is effective to fit clutter power spectrum. The model establishes quantitative relationship between spectral characteristics of reverberation chamber and stirring speed and signal frequency, meanwhile it builds a bridge between $\mathrm{RC}$ and clutter.

However, the above model cannot fit the spectrum of the RC accurately, the model can be effective by modifying the above model:

$$
P(f)=\mathrm{a} \frac{\lambda \beta}{4} \exp \left(-\frac{\lambda \beta}{2}|f|\right)+k,-\infty<f<\infty
$$

Where $\lambda$ depends on frequency and $\beta$ depends on stirring 
speed, $\mathrm{k}$ is constant. Compared to the above clutter model, it is found that the above-mentioned models are only the variation of the coefficients, which means that the clutter environment is similar to the reverberation chamber environment.

\section{Measurement}

Measurements are accomplished by using the $\mathrm{RC}$ present at Mechanical Engineering College that is of a $10.5 m \times 7.9 m \times 4.3 m$ volume where two stirrers (horizontal and vertical) realize the randomization of the input signal to realize field environment with the characteristic of statistically homogeneous, isotropic, random polarization. A photo of the measurement setup is shown in Figure 1 and Figure2.

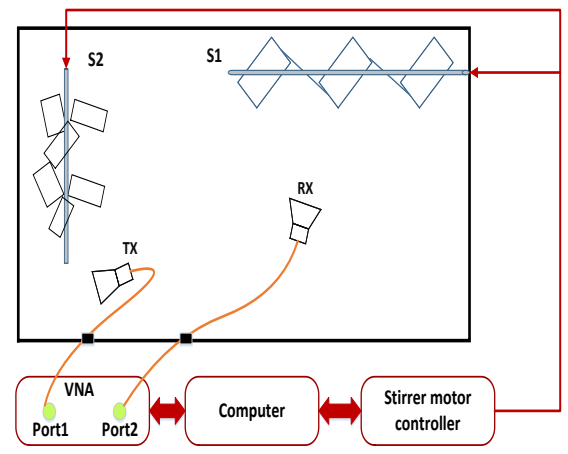

Figure 1: schematics measurement setup

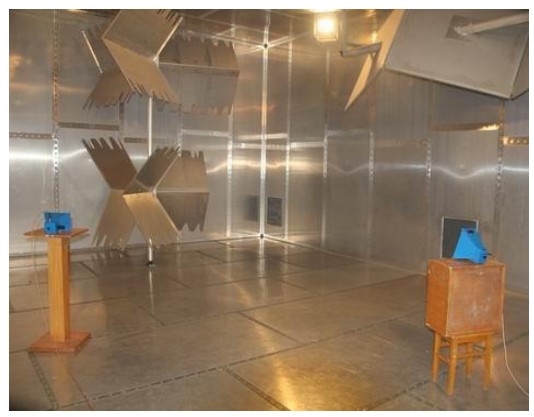

Figure 2: measurement setup in the $\mathrm{RC}$ at Mechanical Engineering College

In this measurement, transmit antenna point to a corner and received antenna point to stirrer S2 inside effective working area to reduce the dc part. The vertical stirrer S2 work with velocity $10 \mathrm{rpms}$ and measurements are implemented at different frequencies ( $1 \mathrm{GHz}$ and $200 \mathrm{MHz}$ respectively) to fit the model for different frequencies. A two-port Vector Network Analyzer (VNA) is used to collect data and a simple single frequency at the two above-mentioned frequencies is transmitted through the chambers and 1601 samples are acquired.

The scattering coefficient S21 is measured which will be used to analyze power spectrum. The two groups of pictures are the result of fitting the experimental data and the model. The Figure 3 and the Figure 4 represent the result of measurement when the frequency is $1 \mathrm{Ghz}$ and $200 \mathrm{MHz}$ respectively. Note that the zero frequency corresponds to the input frequency that is in such a case $1 \mathrm{GHz}$ and $200 \mathrm{MHz}$.

The result shows a good fit between the two curves and the phenomenon the wider of the Doppler spread, the higher of the frequency is validated.

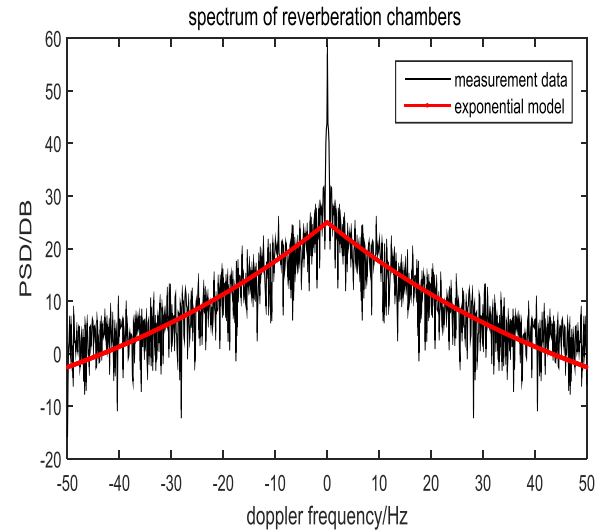

Figure 3: Spectrum of the $\mathrm{RC}$ in $1 \mathrm{GHz}$

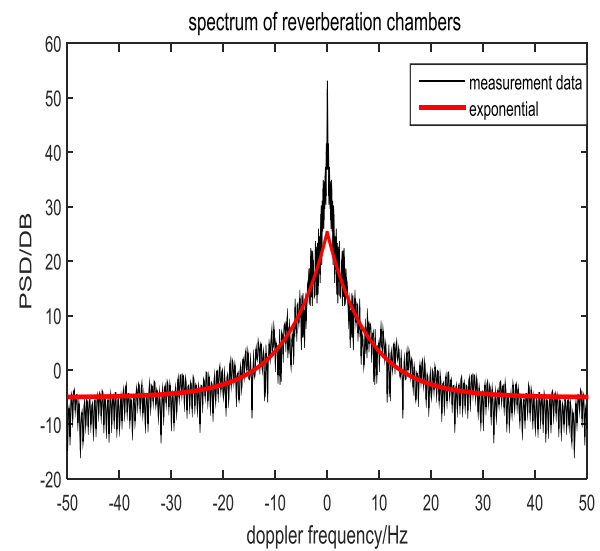

Figure 4: Spectrum of the RC in $200 \mathrm{MHz}$

However, the model is not good fit to the measurement data near zero frequency obviously because of the more powerful dc component. Although the transmit antenna point to a 
corner and received antenna point to stirrer S2, it is not all signals will be stirred by stirrer S2, there remains a part of energy which is received without being stirred. For the purposes of decreasing the effect of the dc component for better fitting, making the transmit antenna point to stirrer $\mathrm{S} 2$, meanwhile, the transmitting and receiving antennas are cross-polarized.

\section{Conclusion}

In this paper, the reverberation chamber is introduced to simulate radar clutter for the first time. The similarity between reverberation chamber field environment and radar clutter environment and the feasibility of simulating radar clutter environment in reverberation chamber are analyzed. A novel spectrum model which is proposed and show a good agreement to the measurement data. It indicates it is possible to simulate the Doppler spectrum of radar clutter in RC, furthermore, it is verified that frequency is proportional to the Doppler spread. The future work should concentrate on exploring the quantitative relationship between the Doppler spread and frequency.

\section{References}

[1] Lombardo, P., and Billingsley, J. B. (1999) A new model for the Doppler spectrum of windblown radar ground clutter. In Proceedings of the IEEE 1999 Radar Conference, Boston, Apr. 1999, 142-147.

[2] Lei Chang. Simulation and application of radar clutter [D]. Xian Electronic Science \& Technology University, 2010.

[3] Jianjin Ding. Theory, measurement and design of reverberation chamber [D]. Beijing Jiaotong University, 2005.

[4] Ramiro Serra, Flavio Canavero, Field statistics in a one-dimensional Reverberation Chamber model, Comptes Rendus Physique, Volume 10, Issue 1, January.2009,Pages 31-41.

[5] D. A. Hill, "Plane wave integral representation for fields in reverberation chambers," in IEEE Transactions on Electromagnetic Compatibility, vol. 40, no. 3, pp. 209-217, Aug 1998.
[6] D. A. Hill, "Uniqueness of plane wave integral representation for idealized fields in reverberation chambers," IEEE Trans. EMC, vol. 57, no. 3, pp. 584-586, June 2015.

[7] Hao Wang, Zhangzhi Han, Qiang He, Jiandong Ga o. Simulation of radar clutter Doppler spectrum ba sed on reverberation chamber[J]. Modern radar,201 3, (09):69-72.

[8] Fishbein, W., Graveline, S. W., and Rittenbach, O. E. (1978) Clutter attenuation analysis. TR ECOM-2808, Fort Monmouth, NJ, US Army Electronics Command, Mar. 1967. Reprinted in D. C. Schleher (Ed.), MTI Radar, Boston, MA: Artech House, 1978.

[9] Q. Xu, Y. Huang, L. Xing, Z. Tian, M. Stanley and S. Yuan, "B-Scan in a Reverberation Chamber," in IEEE Transactions on Antennas and Propagation, vol. 64, no. 5, pp. 1740-1750, May 2016. 